\title{
Crystal Fields in Dilute Alloys of RareEarths in Sc, Y, and Lu Studied by Neutron
} Scattering

\section{Rathmann, Ole; Touborg, Pernille Høybye}

\section{Published in:}

Physical Review B

Link to article, DOI:

10.1103/PhysRevB.16.1212

Publication date:

1977

Document Version

Publisher's PDF, also known as Version of record

Link back to DTU Orbit

Citation (APA):

Rathmann, O., \& Touborg, P. H. (1977). Crystal Fields in Dilute Alloys of RareEarths in Sc, Y, and Lu Studied by Neutron Scattering. Physical Review B, 16(3), 1212-1216. https://doi.org/10.1103/PhysRevB.16.1212

\section{General rights}

Copyright and moral rights for the publications made accessible in the public portal are retained by the authors and/or other copyright owners and it is a condition of accessing publications that users recognise and abide by the legal requirements associated with these rights.

- Users may download and print one copy of any publication from the public portal for the purpose of private study or research.

- You may not further distribute the material or use it for any profit-making activity or commercial gain

- You may freely distribute the URL identifying the publication in the public portal 


\title{
Crystal fields in dilute alloys of rare earths in Sc, Y, and Lu studied by neutron scattering
}

\author{
O. Rathmann \\ Atomic Energy Commission Research Establishment Risø, DK-4000 Roskilde, Denmark \\ P. Touborg \\ Department of Electrophysics, Technical University of Denmark, DK-2800 Lyngby, Denmark \\ and Physics Department, University of Odense, DK-5000 Odense, Denmark
}

(Received 7 April 1976; revised manuscript received 31 March 1977)

\begin{abstract}
The crystal-field levels in Sc-Er, Lu-Er, and Y-Dy have been studied by neutron spectroscopy. The spectra for $\mathrm{Sc}-\mathrm{Er}$ and $\mathrm{Lu}-\mathrm{Er}$ are similar to earlier published spectra for $\mathrm{Y}-\mathrm{Er}$ and in agreement with the level schemes deduced from bulk magnetization measurements. Disagreements between the two types of measurement for Y-Dy suggest a correction for magnetic ordering in the analysis of the magnetization data. The reanalyzed magnetization results are fully consistent with the neutron results.
\end{abstract}

\section{INTRODUCTION}

The present paper reports on inelastic neutron scattering experiments on Sc-2-at.\% Er, Lu-2at. \% Er, and Y-1-at.\% Dy. These measurements and earlier published measurements ${ }^{1}$ on $\mathrm{Y}-2$-at. \% Er were performed to provide an independent check on crystal-field parameters deduced from bulk magnetization measurements. ${ }^{2-5}$ With the above chosen alloys the variation of crystal-field parameters both with the rare-earth solute and with the host can be studied. The present neutron scattering experiments have revealed errors in the published crystal-field parameters for $\mathrm{Y}-\mathrm{Dy} .^{2,3}$ These errors are due to the effects of magnetic ordering on the zero-field susceptibility which was used to derive the crystal-field parameters.

We demonstrate a method for correction of these effects which give crystal-field parameters consistent with the neutron results. This method can be used for all the heavy rare earths diluted in $\mathrm{Y}$, $\mathrm{Lu}$, or Sc hosts and has been employed in the preceeding paper.

\section{EXPERIMENTAL TECHNIQUE}

Single crystals of the alloys Sc-2.0-at.\% Er, Y-3.0-at. \% Dy, Y-1.1-at. \% Dy, and Lu-2.0 at.\% Er were grown using the recrystallization method described in Ref. 6. The chosen shape and size (typically $2 \mathrm{~cm}^{3}$ ) of the samples were determined from considerations of neutron absorption. Some of the samples consisted of two single crystals aligned with the same orientation. All samples were studied by neutron diffraction, to look for possible ordering, and by inelastic neutron scattering to observe transitions between crystal-field levels. In the latter experiments the intensity spectrum of scattered neutrons with fixed wavevector transfer $(\kappa)$ and fixed incident energy was measured on a triple-axis spectrometer with a counting time determined by a preset number of beam monitor counts (BMC). The incident energy was generally $14.4 \mathrm{meV}$ and graphite filter was used to remove second-order contamination. The energy resolution was about $1 \mathrm{meV}$. In one case a $5-\mathrm{meV}$ incident energy and a Be filter cooled to 77 $\mathrm{K}$ were used to obtain a resolution of $0.2 \mathrm{meV}$. The measurements were performed at temperatures down to $5 \mathrm{~K}$ using a cryostat with $\mathrm{Al}$ windows.

\section{EXPERIMENTAL RESULTS}

No ordering was observed at temperatures above $5 \mathrm{~K}$ in Sc-2.0-at.\% Er, Y-1.1-at.\% Dy, and $\mathrm{Y}-$ 2.0 -at. \% Er. In $\mathrm{Y}-3.0$-at.\% Dy helical ordering with the moments lying in the basal plane was observed below $9 \mathrm{~K}$. The neutron diffraction scan is illustrated in Fig. 1. The spiral angle is $50^{\circ}$ per layer. Inelastic scans above the ordering temperature showed no sign of crystal-field transitions, probably due to the large exchange interactions responsible for the ordering. Also in Lu-2.0-at.\% Er magnetic satellites were present. The corresponding order existed at least up to $20 \mathrm{~K}$, indicating that the ordering takes place in small regions of high Er concentration. An estimate from satellite intensities showed that less than $3 \%$ of the Er takes part in the ordering. Thus the ordering may be neglected in interpreting the inelastic scattering experiment.

The neutron scattering intensity spectrum for Sc-2.0-at.\% Er is shown in Fig. 2(a). Two transitions, indicated by arrows, are observed. The transition energies determined by a least-squares fit of a sum of Gaussians to the spectra are shown in Fig. 2(b). These results are in agreement with the earlier published crystal-field parameters determined from analysis of magnetization measure- 


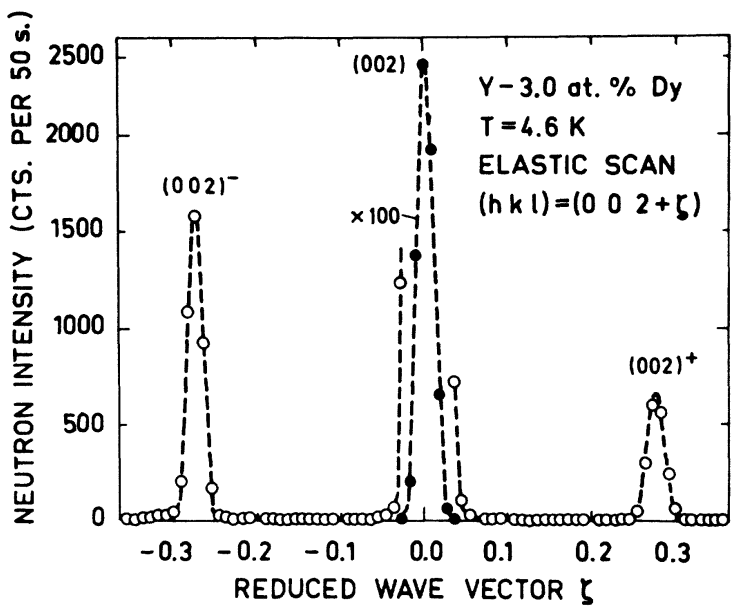

FIG. 1. Neutron-diffraction scan for $Y-3.0$-at. \% Dy along the [002] direction. The satellites $(002)^{ \pm}$are not present above $9 \mathrm{~K}$.

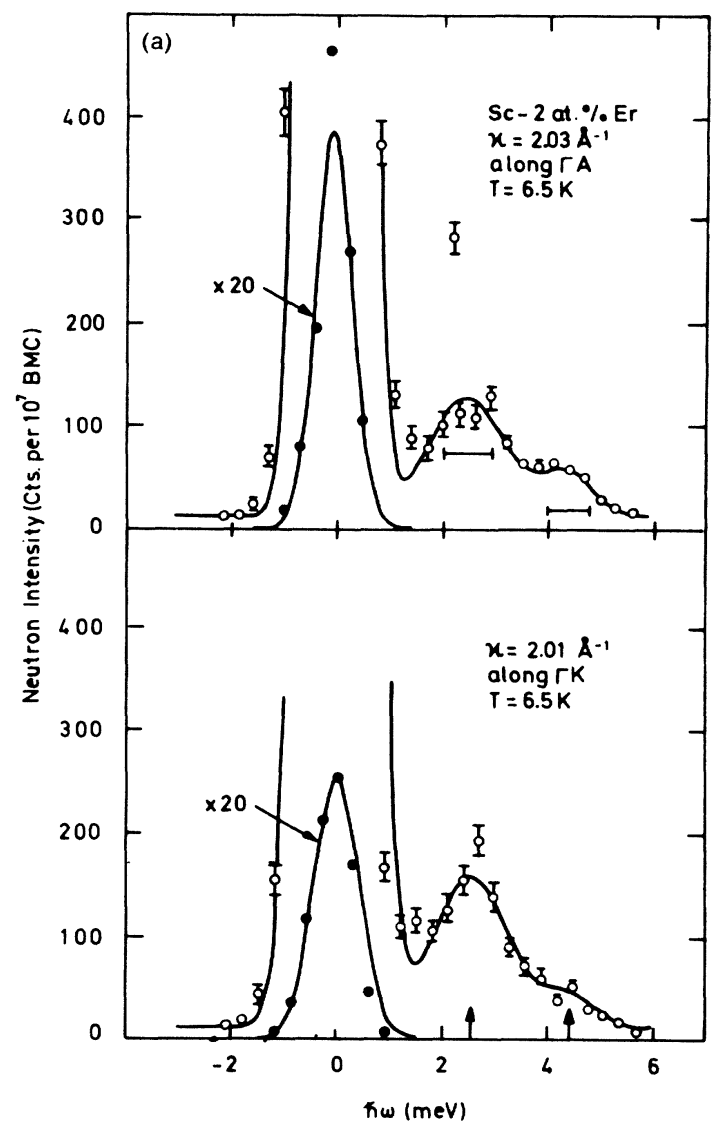

ments..$^{5}$

At the temperature $6.5 \mathrm{~K}$ of the neutron experiment only the crystal-field ground level is populated. Thus only transitions from this level to excited levels are observed. Measurements at higher temperatures or with larger wave-vector transfer give the same spectrum as Fig. 2(a) but with decreased intensities of the transition peaks. This behavior, also seen for the other samples, is in agreement with what is expected for pure crystalfield transitions.

The spectra for Lu-2.0-at.\% Er, Fig. 3(a), show one significant peak (arrow at lower energy) and contain evidence of another (arrow at higher energy). Also for Lu-2.0-at.\% Er the transition energies, Fig. 3(b), agree with the earlier published crystal-field parameters. ${ }^{4}$

The spectra at $5 \mathrm{~K}$ for $\mathrm{Y}-1.1$-at. \% Dy show one transition shoulder [arrow in Fig. 4(a)]. The iden-

FIG. 2. (a) Inelastic neutron scattering spectra for Sc-2.0-at.\% Er. The experimental resolution and statistical uncertainties are indicated. The full lines are least-squares fits. (b) Transition energies with uncertainties obtained from the spectra in (a). (c) Crystal-field levels and wave functions calculated from the average crystal-field parameters in $K$ for Sc host (Table II in preceeding paper): $B_{20} / \alpha=-29.5 \pm 0.9, B_{40} / \beta=9.9 \pm 1.9, B_{60} / \gamma=19.8 \pm 1.5, B_{66}=\frac{77}{8} B_{60}$ The uncertainties in energy of the lowest levels and observable transitions at zero temperatures are indicated. 

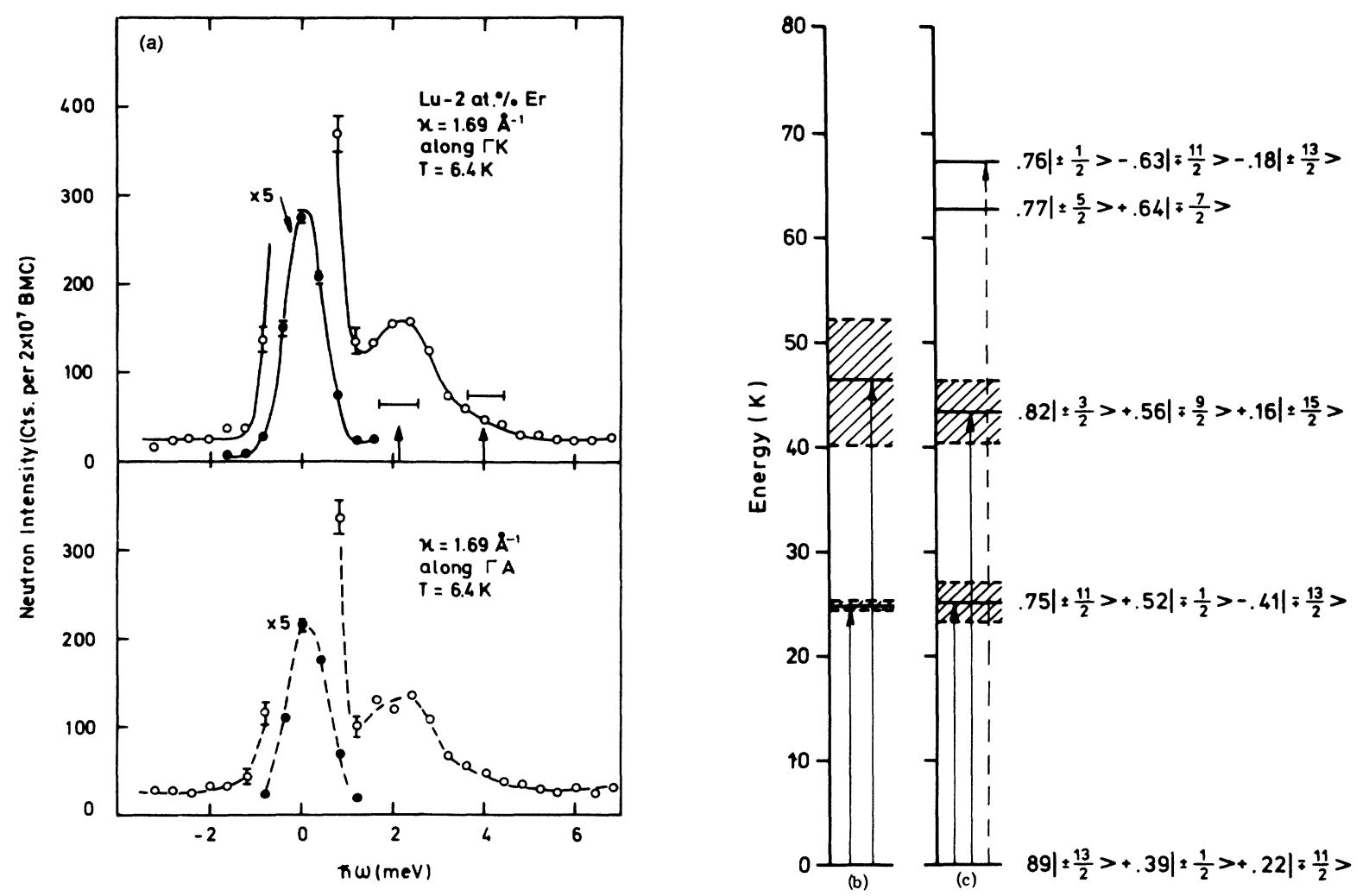

FIG. 3. (a) Inelastic neutron spectra for Lu-2.0-at\% Er. Resolution and uncertainties are indicated. The full line is a least-squares fit. The dashed line is a guide to the eye. (b) Transition energies with uncertainties obtained from the spectra in (a). (c) Crystal-field levels and wave functions calculated from the average crystal-field parameters in $\mathrm{K}$ for Lu host (Table II in preceeding paper): $B_{20} / \alpha=-53.4 \pm 2.3, B_{40} / \beta=6.5 \pm 1.6, B_{60} / \gamma=14.1 \pm 1.2, B_{66}=\frac{77}{8} B_{60}$. The uncertainties in energy of the lowest levels are indicated. The dashed arrow indicates a possible transition which has too small intensity to be observed experimentally.

tification of the shoulder as a crystal-field transition is supported by the decrease in intensity at 30 $\mathrm{K}$ also shown in Fig. 4(a). An attempt to resolve the transition peak completely by using an energy resolution of $0.2 \mathrm{meV}$ was unsuccessful, because it has an inherent width of about $1 \mathrm{meV}$. The transition energy $1.33 \pm 0.2 \mathrm{meV}$ [Fig. 4 (b)] is in disagreement with the first-excited crystal-field level of $3.7 \mathrm{meV}$ as calculated from earlier deduced parameters (Fig. 12 in Ref. 2). However the knowledge that ordering effects are present has made it possible to correct the magnetization data for these effects as described below.

When this correction is applied to the magnetization data for all the dilute heavy rare earths ( $\mathrm{Tb}$ to $\mathrm{Tm}$ ), the deduced crystal-field parameters $B_{l m} / \alpha_{1}$ are, for each of the diluents Sc, Y, and $\mathrm{Lu}$, sufficiently close to be represented by a single set of average parameters (see preceeding paper). As shown by parts (b) and (c) of Figs. 2-4 these sets of average crystal-field parameters are in excellent agreement with the present inelastic neutron scattering results.

\section{REANALYSIS OF THE MAGNETIZATION MEASUREMENTS}

The crystal-field parameters $B_{20} / \alpha, B_{40} / \beta, B_{60} /$ $\gamma$, and $B_{66} / \gamma$, the two exchange parameters $\gamma_{\|}$and $\gamma_{\perp}$, and the concentration are determined by fits to the inverse initial susceptibility. The fitting procedure is described in the preceding paper. Effects of ordering even in the dilute alloy $\mathrm{Y}-$ $0.137-$ at. \% Dy are clearly seen in the measurements of the basal-plane anisotropy. ${ }^{3}$ The presence of a small ordered region may change the initial susceptibility drastically. However, the correct paramagnetic susceptibility can in the important cases be obtained from isothermal magnetization curves. For an applied field of sufficient magnitude $\left(H>H_{\mathrm{min}}\right)$ all moments in the crystal will be aligned in the field direction, although acted on by 

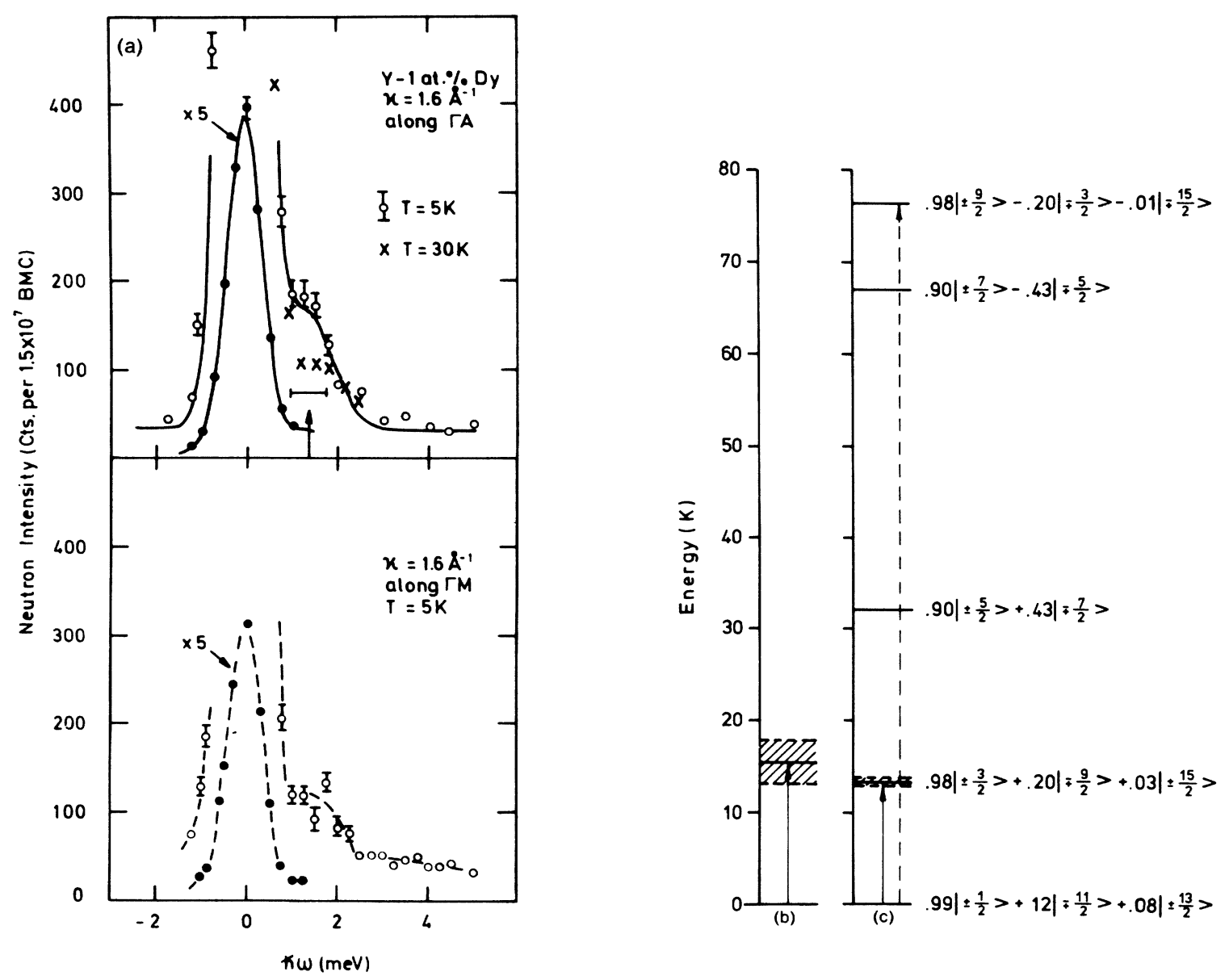

FIG. 4. (a) Inelastic neutron spectra for Y-1.1-at. \% Dy. Resolution and uncertainties are indicated. The full line is a least-squares fit to the spectrum at $5 \mathrm{~K}$. The dashed line is a guide to the eye. (b) Transition energy with uncertainties obtained from the spectrum in (a). (c) Crystal-field levels and wave functions calculated from the average crystalfield parameters in $\mathrm{K}$ for $\mathrm{Y}$ host (Table $\Pi$ in preceding paper): $B_{20} / \alpha=-102.7 \pm 5.7, B_{40} / \beta=7.1 \pm 1.2, B_{60} / \gamma=12.2 \pm 0.4$, $B_{66}=\frac{77}{8} B_{60}$. The uncertainties in energy of the lowest level is indicated. The dashed arrow indicates a possible transition which has too small intensity to be observed experimentally.

different exchange fields dependent on the local concentration. If the sum of the exchange and the applied fields is within the range for which the pure crystal-field susceptibility $\chi_{C F}$ remains at its initial value, we find the following formulas for the differential susceptibility in the molecularfield approximation:

$$
\begin{aligned}
& M_{j}=\left(H+\gamma_{j}\langle M\rangle \chi_{\mathrm{CF}},\langle M\rangle=\frac{1}{N} \sum_{j=1}^{N} M_{j},\right. \\
& \frac{1}{\chi}=\left.\frac{d H}{d\langle M\rangle}\right|_{H H_{\min }}=\frac{1}{\chi_{\mathrm{CF}}}-\langle\gamma\rangle,\langle\gamma\rangle=\frac{1}{N} \sum_{j=1}^{N} \gamma_{j} .
\end{aligned}
$$

Ths sums are over sites $j, \gamma_{j}$ are site-dependent exchange constants and $M_{j}$ are the magnetic moments. The only difference of (1) from the formulas for an ideal dilute system is the change of $\gamma$ to

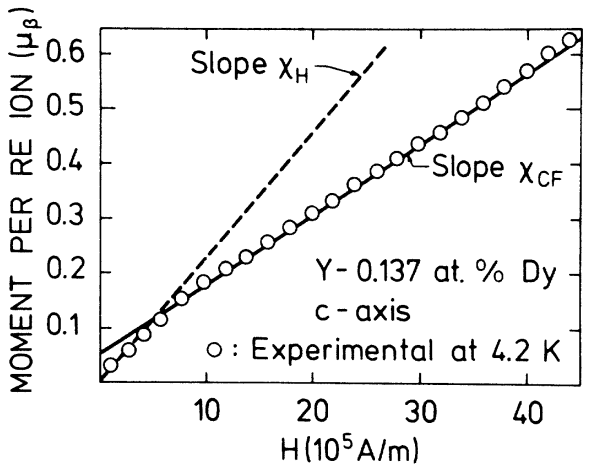

FIG. 5. Magnetization at $T=4.2 \mathrm{~K}$ for $\mathrm{Y}-0.14-$ at. $\%$ Dy in the $c$ direction. The experimental differential slope at higher fields, $\chi_{C F}$ (slope of full line), and the earlier published susceptibility $\chi_{H}=H / M$ at $H=1.35 \times 10^{5} \mathrm{~A} / \mathrm{m}$ (slope of dashed line) are illustrated. 


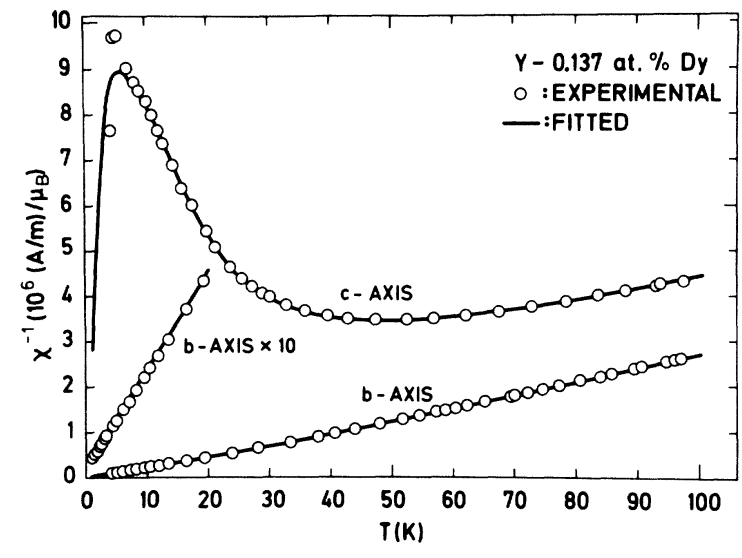

FIG. 6. Reciprocal susceptibility for Y-0.14-at.\% Dy. The experimental points are published data (Ref. 2) corrected in the $c$ direction as described in the text. The full curves are theoretical best fits, giving the parameters $B_{20} / \alpha=-115 \pm 12 \mathrm{~K}, B_{40} / \beta=7.6 \pm 3.5 \mathrm{~K}, B_{60} / \gamma=13.1$ $\pm 3 \mathrm{~K}, B_{66}=\frac{77}{8} B_{60}, \gamma_{11}=(-0.3 \pm 0.1) \times 10^{6}(\mathrm{~A} / \mathrm{m}) / \mu_{B}, \gamma_{\perp} \approx 0$.

$\langle\gamma\rangle$. The existence of an extended linear range in the magnetically hard directions may be verified theoretically after the parameters have been deduced. [For $\mathrm{Y}-\mathrm{Dy} \chi_{\mathrm{CF}}$ varies less than $2 \%$ in the range $\left(0-70 \times 10^{5} \mathrm{~A} / \mathrm{m}\right.$.]

The validity of the above theory can be verified experimentally by the presence, in the magnetization curves, of a linear range following the initial high slope range. This feature is clearly seen in the magnetization curve shown in Fig. 5. In Fig. 5 is illustrated the difference at $4.2 \mathrm{~K}$ between $\chi_{C F}$ and the earlier published susceptibility $\chi_{H}=M / H$ measured at the constant field $H=1.35 \times 10^{5} \mathrm{~A} / \mathrm{m}$. $\chi_{C F}$ equals $\chi_{H}$ for Y-0.137-at.\% Dy at temperatures above $13 \mathrm{~K}$. For Er alloys $\chi_{C F}$ equals $\chi_{H}$ in the whole measuring range.

For all alloys measured (see preceding paper), the above procedure applies in the hard direction down to $4 \mathrm{~K}$. In the easy directions at low temperature, the initial field-independent range of $\chi_{C F}$ is too narrow to allow the above procedure. Fortunately the susceptibilities at low temperatures in the easy directions are rather unimportant in determining the parameters.

For $Y-0.137-a t . \%$ Dy $\chi_{C F}$ has been determined from a number of isothermal magnetization curves, and the earlier published $1 / \chi$ curves have been corrected. The corrected curves are shown in Fig. 6 with the new theoretical best fits.

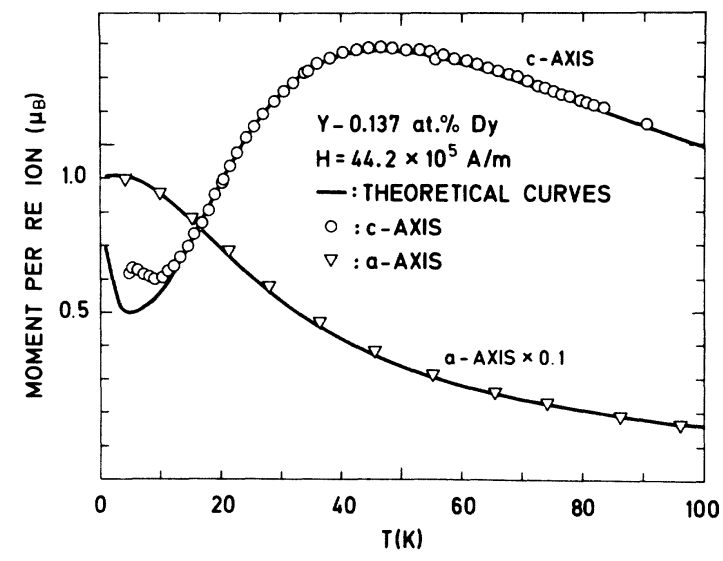

FIG. 7. Isofield curves at $44.5 \times 10^{5} \mathrm{~A} / \mathrm{m}$ for $\mathrm{Y}-0.14-$ at.\% Dy. The experimental points were published earlier (Ref. 2). The full curves are calculated using the parameters given in the caption to Fig. 6.

For a further check of the revised crystal-field parameters for $\mathrm{Y}-\mathrm{Dy}$, they were compared to magnetization measurements at higher fields. Figure 7 shows isofield measurements at 44.2 $\times 10^{5} \mathrm{~A} / \mathrm{m}$ for $\mathrm{Y}-0.137$-at. \% Dy, ${ }^{2}$ together with theoretical curves. The deviation at low temperatures in the $c$ direction is expected due to the ordering mentioned earlier. Also magnetization measurements up to $300 \times 10^{5} \mathrm{~A} / \mathrm{m}$ are in agreement with the theoretical predictions (Fig. 7 in preceding paper).

Ordering effects in the Er alloys are too small to give any corrections to the initial susceptibility. This is consistent with the agreement between the magnetization and neutron results for these.

\section{CONCLUSION}

The neutron experiments have demonstrated the necessity of corrections for ordering effects in some dilute alloys. The crystal-field parameters obtained from the magnetization data, corrected if necessary in the way described, are in accordance with the results of the independent and direct method of neutron scattering.

\section{ACKNOWLEDGMENTS}

The authors appreciate fruitful discussions with J. Als-Nielsen, A. R. Mackintosh, J. Høg, and V. Frank.
${ }^{1}$ O. Rathmann, J. Als-Nielsen, P. Bak, J. Hog, and P. Touborg, Phys. Rev. B 10, 3983 (1974).

${ }^{2} \mathrm{~J}$. Hog and P. Touborg, Phys. Rev. B 9, 2920 (1974).

${ }^{3} \mathrm{~J}$. Hog and P. Touborg, Phys. Rev. B $\underline{11}, 520$ (1975).
${ }^{4}$ P. Touborg and J. Hog, Phys. Rev. B 11, 2660 (1975).

${ }^{5} \mathrm{~J}$. Høg and P. Touborg, Phys. Rev. B 14,1209 (1976).

${ }^{6} \mathrm{~K}$. A. McEven and P. Touborg, J. Phys. F 3,1903 (1973). 